\title{
La sociologie et les régimes de réalité
}

Sociology and the Regimes of Reality

La sociología y los regímenes de realidad Colocando las

relaciones entre la acción y la realidad en el centro de la teoría social, el artículo se propone explorar las nuevas orientaciones posibles para el trabajo de la sociología

\section{Danilo Martuccelli}

Numéro 59-60, automne 2015, hiver 2016

Les nouveaux objets de la sociologie

URI : https://id.erudit.org/iderudit/1036788ar

DOI : https://doi.org/10.7202/1036788ar

\section{Aller au sommaire du numéro}

\section{Éditeur(s)}

Athéna éditions

\section{ISSN}

0831-1048 (imprimé)

1923-5771 (numérique)

Découvrir la revue

\section{Citer cet article}

Martuccelli, D. (2015). La sociologie et les régimes de réalité. Cahiers de recherche sociologique, (59-60), 99-112. https://doi.org/10.7202/1036788ar
Résumé de l'article

En mettant les relations entre l'action et la réalité au centre de la théorie sociale, l'article propose d'explorer de nouvelles orientations possibles pour le travail sociologique. L'interrogation première de la sociologie doit porter sur un monde social où, quelle que soit la force des coercitions, il est toujours possible d'agir autrement. Face à l'élasticité foncière de la vie sociale, et afin de contrer le vertige suscité par l'illimitation de l'action, les sociétés ont institué différentes limites indépassables de la réalité. Quatre grands domaines donnant forme à différents régimes de réalité ont historiquement rempli cette fonction (la religion, la politique, l'économie, l'écologie), en articulant présupposés imaginaires et expériences effectives. Dans les sociétés contemporaines, c'est à l'économie, véritable régime hégémonique de réalité, et en partie à l'écologie, que reviennent cette fonction. La reconnaissance des spécificités de cette dimension ouvre à de nouvelles problématiques sociologiques. 


\section{La sociologie et les régimes de réalité}

Danilo Martuccelli

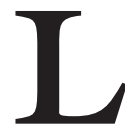

a question première de la sociologie est souvent associée au problème de l'ordre social et à la formulation canonique qu'en a donnée Tacott Parsons: comment l'ordre social est-il possible ${ }^{1}$ ? Pourtant, il est possible de poser une autre interrogation, tout aussi fondamentale, à la base de la théorie sociale - l'étude effective des manières dont la vie sociale contraint et habilite les acteurs. Étrangement, cette question, pourtant au cour du travail sociologique, n'a vraiment reçu une attention théorique majeure que depuis quelques décennies autour de l'agency. Cependant, dans ces études, les coercitions, comme principales manifestations de la résistance de la réalité sociale, ont toutefois souvent été mobilisées de manière assez imprécise.

Pour le comprendre, partons de Durkheim et de sa caractérisation des faits sociaux - des phénomènes caractérisés par leur objectivité, leur extériorité et leur sanction ${ }^{2}$. Ces trois aspects sont pourtant loin d'être évidents. L'idée que les faits sociaux, en eux-mêmes, et indépendamment des croyances et des actions individuelles, puissent exercer des contraintes sur les acteurs est une proposition polémique. Et pourtant, en dépit de cette hypostase en partie illégitime, il y a quelque chose de foncièrement juste dans la vision durkheimienne, lorsqu'elle souligne l'extériorité des contraintes que la vie sociale oppose aux acteurs. Par "contrainte», Durkheim désignait donc tour à tour les obstacles que le monde externe impose aux acteurs dans la

1. Talcott Parsons, The Structure of Social Action, Glencoe, The Free Press, 1949 [1937].

2. Émile Durkheim, Les règles de la méthode sociologique, Paris, PUF, 1987 [1895]. 
poursuite de leurs objectifs, les effets de l'environnement social (notamment de nature morphologique) sur les actions, les sanctions qu'un groupe social est capable d'imposer aux membres qui ne respecteraient pas les normes, la force de l'autorité ou des règles morales intériorisées lors de la socialisation. En fait, lorsque Durkheim parle de «contraintes», il pense fréquemment influence, et même influence légitime.

La réalité de la vie sociale a donc été bâtie par la sociologie autour d'une série de contraintes. Pourtant, et c'est ce qui nous intéresse de souligner ici, chez Durkheim, la différenciation établie entre les diverses coercitions (morphologiques, morales) s'estompe devant leur grande similitude fonctionnelle: toutes les contraintes sont représentées comme imposant une limite plus ou moins indépassable et évidente aux acteurs ${ }^{3}$. Le social est conçu comme un niveau d'agencement particulier de la réalité, soumis aux conditionnements de courants, de forces, de contraintes dont le travail effectif reste cependant souvent opaque. La question des coercitions devient une affaire d'inférence logique ou de corrélation statistique: ce qui laisse souvent sous-analysé le problème concret et effectif du travail des contraintes. Or, dans la vie sociale, les contraintes opèrent de façon irrégulière (elles peuvent, en fonction de certains contextes, ne pas agir): intermittente ou médiate (toute contrainte se déroule dans un temps plus ou moins long - ce qui complexifie l'idée de la réactivité de l'environnement) ${ }^{4}$ : transitoire (les sanctions évoluent, se transforment, se durcissent ou disparaissent).

En partant de ce «point aveugle» de la théorie sociale, dans ce qui suit, nous nous efforcerons de montrer comment une étude détaillée de cette problématique peut être à la fois un nouvel objet d'étude ouvrant à de nouvelles orientations analytiques et critiques.

\section{L'action et la réalité}

Si l'on interroge la réalité des faits sociaux, non plus à partir de la mesure objective des contraintes, mais à partir de l'expérience des sanctions éprouvées par les acteurs, la problématisation de base de la théorie sociale change quelque peu de nature. Une autre question s'impose: le double constat de l'évidence d'un monde social supposé contraindre fortement les conduites et le caractère souvent hautement problématique (non direct, non immédiat) des sanctions effectives sur les actions. L'interrogation première de la théorie sociale se déplace ainsi vers les caractéristiques que doit posséder la réalité

3. Dans un sens proche, Anthony Giddens, La constitution de la société, Paris, PUF, 1987 [1984].

4. Un point fort bien établi par les travaux du réalisme critique, Margaret Archer (dir.), Critical Realism. Essential Readings, Londres, Routledge, 1998. 
sociale pour que l'action y soit toujours possible. La question centrale devient la possibilité irréductible de l'action - dans un monde social où, quelle que soit la force des coercitions, il est toujours possible d'agir autrement. Cet «autrement» est à comprendre en rapport à une norme dominante ou à une fréquence statistique.

Dans chaque situation, l'acteur fait l'expérience concrète d'un spectre de possibilités qui s'ouvre à lui. Certes, les actions ne sont ni aléatoires ni imprévisibles (elles passent toujours par des orientations culturelles partagées), mais elles ne sont soumises à aucune nécessité irrécusable. La conduite n'est pas incertaine mais contingente. Mais cette contingence ne dépend pas principalement de la liberté, de l'agency, des capacités corporelles, cognitives ou stratégiques des individus ${ }^{5}$. Il faut prendre des distances face à ces conceptions de l'action. Tant que l'on n'abandonne pas cette représentation, la tentation est grande de trouver la nouveauté dans l'histoire, en dernière instance, au seul niveau de la créativité des acteurs. Bien entendu, les individus ont une force inventive propre. Mais une chose est d'en saisir le rôle à partir du différentiel de possibilités offertes par la vie sociale, et une autre est de la cerner à partir des compétences cognitives ou en termes de liberté. Une ligne de partage sépare ainsi les travaux de psychologie sociale (où l'accent est mis sur les capacités des acteurs) et les réflexions sur l'ontologie de la vie sociale (où, tout en partant de l'action il est question de comprendre le mode opératoire des contraintes de l'environnement).

La question première de la théorie sociale est ainsi de comprendre un univers social où un nombre important et différent d'actions, au moins momentanément, est toujours simultanément possible. C'est la consistance particulière de la vie sociale qui est toujours - et partout - à la source de cette possibilité d'agir autrement. Tout en s'accordant sur le postulat que la réalité est ce qui résiste, il s'agit donc d'attirer l'attention sur les moments de problématisation effective de l'action par les sanctions de la réalité sociale.

Il s'agit d'une question déjà en partie abordée par le pragmatisme et sa volonté de faire de la réussite ou de l'échec d'une action son critère central d'évaluation ${ }^{7}$. Les sanctions de la réalité seraient donc à étudier en aval de l'action. Un des grands mérites du pragmatisme est justement d'avoir souligné à quel point l'acteur est dépendant, lors de l'évaluation de la réussite ou

5. Jean-Paul Sartre, L'être et le néant, Paris, Gallimard, 1943: Alain Touraine, Production de la société, Paris, Seuil, 1973: Cornelius Castoriadis, L'institution imaginaire de la société, Paris, Seuil, 1975: Hans Joas, La créativité de l'agir, Paris, Cerf, 1999 [1992] : Franco Crespi, Teoria dell'agire sociale, Bologne, II Mulino, 1999.

6. Danilo Martuccelli, La consistance du social, Rennes, PUR, 2005.

7. William James, Le pragmatisme, Paris, Flammarion, 2007 [1907]. 
de l'échec de son action, du contexte interindividuel et collectif dans lequel il agit (les clés de la réussite ou de l'échec d'une action ne sont jamais immédiatement données par un état matériel du monde: les raisons et les facteurs de réussite et d'échec se construisent dans le cadre des relations sociales). En prenant cette position, les pragmatistes ne récusent pas l'existence d'une réalité extérieure aux acteurs, mais ils soulignent que l'action de l'environnement est indissociable des significations sociales. Or, le succès d'une action, ici et maintenant, ne présage en rien de sa fortune future, ce qui questionne en profondeur l'idée d'une sanction-couperet immuable de la réalité et invite à des études précises, autant au niveau de l'action individuelle que collective, sur les processus effectifs de sanction, par la «réalité» des conduites.

C'est justement ce qui pose problème. Les sanctions de la réalité sont loin d'être univoques, que ce soit à propos d'un diagnostic psychiatrique ${ }^{8}$, de l'échec scolaire ${ }^{9}$ ou d'un prix littéraire ${ }^{10}$ : tant les individus peuvent être insensibles aux démentis du monde ${ }^{11}$ : tant ils peuvent appliquer, comme le montre le modèle de la poubelle, n'«importe» quelle solution à différents problèmes $^{12}$ : tant les prophéties autoréalisatrices sont ordinaires ${ }^{13}$. Cette pluralité de figures, que l'on pourrait allonger, invite à enquêter dans une direction complémentaire afin de problématiser la relation entre action et réalité en reconnaissant que, d'ordinaire, le couperet pratique des actions dans la vie sociale repose davantage sur un postulat imaginaire que sur des sanctions concrètes. Autant dire que la certitude des résistances de la réalité s'établit en amont de l'action. Prendre pleinement acte de cette spécificité de la vie sociale oblige non seulement à enquêter, sur les manières dont un acteur, individuel ou collectif, se heurte à une résistance ou à une difficulté effective, mais aussi, voire surtout, à intégrer dans l'analyse la fonction d'un imaginaire qui, en amont de l'action, définit les limites «indépassables» de la réalité en dépit de maintes ouvertures pratiques.

Bien des actions, y compris lorsqu'elles vont à l'encontre de certaines règles ou attentes collectives, ne connaissent pas vraiment de «coups d'arrêt». L'idée d'un environnement social à détente durable et constante, établissant des limites indépassables, et permettant de dégager des accords stables et solides sur ce qu'est la réalité (grâce au succès ou à l'échec des

\footnotetext{
8. Ernst von Glasersfeld, «Introduction à un constructivisme radical», dans Paul Watzlawick (dir.), L'invention de la réalité, Paris, Seuil, 1988 [1981].

9. Anne Barrère, Les lycéens au travail, Paris, PUF, 1997.

10. Nathalie Heinich, L'épreuve de la grandeur, Paris, La Découverte, 1999.

11. León Festinger, Hank Riecken, Stanley Schachter, L'échec d'une prophétie, Paris, PUF, 1993 [1956].

12. Pour une présentation de différents travaux de ce type, James G. March., Johan P. Olsen, Rediscovering Institutions, New York, The Free Press, 1989.

13. Robert K. Merton, Eléments de théorie et de méthodes sociologiques, Paris, Plon, 1965 [1949].
} 
actions), n'est qu'un cas de figure parmi bien d'autres - et loin d'être le plus fréquent. Hormis quelques situations, là où l'échec d'une action, après évaluation et élaboration explicites, prouve pratiquement à l'acteur, comme aux autres, son erreur ${ }^{14}$, l'essentiel de la vie sociale se déroule à l'abri de ce type de considérations. En fait, les individus et les collectifs agissent à partir de limites de la réalité qui, supposées être indépassables, sont transgressées dans les faits sans que cela n'amène à une modification ou à un abandon de la croyance de leur pouvoir de contrainte. Autrement dit: le rapport entre la réalité et l'action ne doit pas être abordé uniquement comme une question se décidant en aval - et tranchée par les conséquences de l'action -, mais comme une certitude postulée en amont et d'emblée par les acteurs.

Pour montrer le glissement de l'aval vers l'amont comme lieu de problématisation du rapport entre l'action et la réalité, évoquons rapidement le Quichotte. La lecture en aval que Schütz en propose est bien connue: comment don Quichotte parvient-il à neutraliser l'existence d'une réalité qui va à l'encontre de ses croyances? La proposition de Schütz est que, pour y parvenir, il est contraint de gérer les contradictions à l'aide d'interprétations ad hoc (comme celle, par exemple, produite par les enchanteurs) permettant la coexistence et la compatibilité entre différents sous-univers de signification ${ }^{15}$. Pourtant, menée à partir de considérations presque exclusivement cognitives, l'analyse de Schütz sous-estime largement les soubassements proprement concrets des aventures du Quichotte, à savoir que les arrangements pratiques avec l'environnement peuvent être multiples et se révéler actifs pendant des laps de temps plus ou moins longs. «N'importe quoi» n'est pas opératoire dans la vie sociale. Et pourtant, bien des attentes, voire des croyances (y compris parmi les plus délirantes, si on se réfère toujours au Quichotte), ne sont en aucun cas immédiatement démenties par le monde.

C'est ce que montre l'analyse de Nabokov qui, en mettant entre parenthèse la vision dominante de la réalité, finit par distinguer vingt actions du Quichotte couronnées de succès et vingt autres sanctionnées par un échec ${ }^{16}$. Certes, une partie de l'explication réside dans des mécanismes de défense proprement cognitifs mobilisés par le personnage de Cervantès. Mais cela n'est qu'une partie de la vérité. Si cela se révèle possible, c'est aussi parce que, très pratiquement, les acteurs peuvent souvent continuer à opérer dans

\footnotetext{
14. Pour l'explicitation de ce postulat pragmatique à partir d'une théorie de l'action, Jürgen Habermas, Vérité et justification, Paris, Gallimard, 2001 [1999].

15. Alfred Schütz «Don Quichotte et le problème de la réalité», dans Essais sur le monde ordinaire, Paris, Le Félin, 2007 [1953]. Cette démarche connaît des voies parallèles dans la psychologie sociale, à commencer par la notion même de dissonance cognitive.

16. Vladimir Nabokov, Littérature III. Don Quichotte, Paris, Fayard, 1992 [1983].
} 
la réalité sociale, avec une efficacité certaine, et pendant un laps de temps plus ou moins long, à l'aide de représentations pourtant jugées comme fausses ou discutables à partir d'une croyance dominante. Les résistances de la réalité sont à cerner autant comme une question imaginaire (en amont donc de toute action et heurt concret) qu'en aval et comme une affaire pratique.

Le propre de la vie sociale tient justement à ce que les individus opèrent au sein d'un environnement où les limites sont fondamentalement indéterminées du point de vue de la pratique et systématiquement présentes au niveau de l'imaginaire. La relation entre l'action et la réalité, pour ouverte qu'elle s'éprouve dans les faits - en aval -, est inséparable - en amont - du postulat de l'existence de moments inévitables et immédiats de corrections.

Poser cette interrogation à la base de la théorie sociale invite à aborder un «nouvel objet» sociologique transversal à bien des domaines et des pratiques: comprendre comment et pourquoi certaines conduites sont opérationnelles et d'autres ne le sont pas, ou ne le sont que transitoirement, mais toujours dans un monde social à malléabilité résistante. Sur ce point, il faut se débarrasser radicalement de l'idée, d'inspiration darwinienne et si constante dans la quasi-totalité des démarches sociologiques, qu'il existe "un » modèle d'adaptation réussie et nécessaire à l'environnement social, qui permettrait de juger, en dernière instance, la fortune de toutes les conduites. Au contraire, il faut reconnaître pour tous les acteurs et à tous les niveaux, que l'élasticité de la vie sociale est une expérience fréquente. Certes, cette élasticité se décline différemment selon les groupes, les contextes ou les trajectoires: les acteurs ont, face à ce différentiel d'élasticité, des marges très différentes d'action, selon qu'ils sont des individus ou de puissants acteurs collectifs. Mais pour tous, et à tous les niveaux, la vie sociale est à la fois indissociablement malléable et résistante. C'est bien cette malléabilité qui rend l'être ensemble continuellement possible. Sans elle, la vie en société serait tout simplement impossible puisqu'elle exigerait un niveau d'articulation rarement atteint dans les pratiques sociales.

\section{Les limites imaginaires de la réalité}

La réalité est ce qui résiste: ce à quoi se heurte l'action. C'est pourquoi la notion qui en rend le mieux compte - du point de vue de la sanction de l'action - est l'idée de choc avec la réalité. Les acteurs vivent en postulant que des limites réelles existent et en croyant surtout qu'elles agissent constamment et plus ou moins immédiatement sur eux. Si le choc avec la réalité mérite la plus grande attention, c'est parce que, continuellement supposé, il est toujours éprouvé de façon complexe. Il est davantage une crainte ima- 
ginaire ou une croyance collective qu'une expérience effective. Et pourtant, son importance est décisive, puisque, en son absence, c'est tout simplement le sens ordinaire de la réalité qui se dissipe. Aucune autre métaphore ne décrit mieux la relation entre l'action et la réalité que celle de l'élasticité, c'est-à-dire, une résistance extensible au cœur d'une limite qui, tout en étant toujours posée, n'est qu'occasionnellement éprouvée.

L'imaginaire des chocs avec la réalité se construit donc au milieu d'une vie sociale dotée d'une élasticité irrépressible. Notons-le: l'ontologie de la vie sociale ouvre à des questions très différentes selon que la réalité est abordée à partir des représentations (dans la plus vénérable tradition philosophique), de sa fabrication (du marxisme au constructivisme) ou de l'action. Ce qui fait problème, chaque fois, est bien différent. En tout cas, aborder la question de l'ontologie de la vie sociale à partir de l'action invite à différencier et à articuler constamment deux aspects. D'une part, reconnaître que l'expérience de l'action est le substrat ordinaire de ce qu'est la réalité. Le sens fondateur de la réalité - ce qui la différencie d'ailleurs du rêve, de la fiction ou de l'hallucination - comporte inévitablement des dimensions de contrainte et de résistance. D'autre part, les contraintes de la réalité sont indissociables du travail d'institution d'un collectif et dans ce sens irréductibles au seul niveau de l'action. Ce qui exige que les analyses en termes de compétences cognitives stricto sensu (et même d'agency) s'ouvrent vers des considérations proprement sociétales - les manières dont les sociétés ont historiquement institué les limites de la réalité.

Les deux niveaux sont différents et ont une autonomie relative. La sociologie peut ainsi analyser les rapports entre l'action et la réalité à l'échelle de l'acteur ou à l'échelle de la société. Ces deux éléments (l'expérience de l'action et le domaine sociétal) sont différents mais c'est bien leur articulation qui structure le sens de la réalité («ce qui résiste») et la fonction qui lui est accordée dans la vie sociale. Tout en abordant la réalité à partir de la question de l'action, dans la mesure où la croyance de la limite de la réalité s'institue toujours en amont des actions, il devient nécessaire tôt ou tard d'élargir la focale au-delà des seules conduites afin de tenir compte des manières dont les sociétés instituent l'imaginaire des grandes limites de la réalité. Autant dire que si l'action rencontre et déjoue toujours des contraintes, la limite, elle, est d'un ordre proprement imaginaire.

Revenons au Quichotte. Le roman de Cervantès décrit une série de chocs avec la réalité et, ne l'oublions pas, d'étonnantes réussites. Ces résultats (surtout les réussites) sont paradoxaux puisqu'ils mettent justement à jour les visions opposées qu'ont le Quichotte et le lecteur des sanctions de la réalité. Pourtant, pour comprendre les étonnements croisés entre le person- 
nage littéraire et le lecteur, il est impossible de rester au seul niveau de l'action. La pleine compréhension du différentiel des résultats n'est intelligible qu'en ouvrant vers les manières historiquement variables dont les sociétés instituent les contraintes de la réalité. Les aventures du Quichotte sont indissociables du passage d'un monde peuplé d'enchanteurs ou des Géants, à un univers social établi sur d'autres critères de réalité - notamment ceux posés progressivement par la science moderne à partir du XVII ${ }^{\mathrm{e}}$ siècle.

Afin de bien souligner le caractère indissociablement historique et institutionnel de ces grandes matrices de représentation, il faut parler de régimes de réalité. Réduite à un schématisme de base, l'histoire de la réalité sociale a été successivement incarnée par quatre grands régimes: la religion, le politique, l'économie, peut-être l'écologie ${ }^{17}$. Toutes les sociétés ont eu historiquement tendance à construire autour d'un domaine d'activité privilégié la limite de la réalité. Chacun de ces domaines, sous des modalités différentes, a ainsi structuré à une période donnée ce qui était censé être le socle ultime de «la» réalité - ce qui dictait l'horizon liminaire du possible et de l'impossible et chaque fois à partir de trois grands éléments. D'abord, l'appel à une expérience immédiate et directe du monde, souvent sous la forme d'un appel au sens commun, dictant comme une évidence sensible irrécusable la force de ce qu'est la «réalité». Ensuite, une production proprement symbolique qui étaye et renforce cette impression - autrement dit: pas d'expérience de la réalité sans un important travail d'élaboration culturelle. Enfin, un ensemble de preuves récurrentes et incontournables, les chocs avec la réalité à proprement parler, qui rappellent, si le besoin se fait sentir, le bien-fondé de ces représentations liminaires du monde.

Chaque régime de réalité désigne à la fois une économie générale du monde - le socle qui soutient tous les autres domaines - et la source ultime du principe de nécessité. La religion d'abord, avec l'action ordinaire des entités invisibles et le partage inviolable du sacré et du profane; puis le politique, en fait l'ordre des hiérarchies naturelles et le besoin du châtiment en cas de leur non-respect; ensuite l'économie, bien sûr, avec la constitution de la rareté et les limites systémiques; et peut-être, déjà, demain l'écologie avec ses interdépendances généralisées et ses seuils indépassables. Chacun de ces domaines, sous des modalités différentes, a structuré, à une période historique donnée, ce qui était censé être le socle ultime de «la» réalité.

Ce «nouvel objet» de théorie sociale - le caractère irrépressible de l'agir autrement - ouvre donc à une «nouvelle thématique» sociologique - la

17. Pour une présentation historique conséquente de cette thèse et de quelques-uns de ses développements, Danilo Martuccelli, Les sociétés et l'impossible, Paris, Armand Colin, 2014. 
question de l'impossible sociétal. Portés par les promesses prométhéennes de la technique ou par le volontarisme du projet de faire l'histoire, les sociétés modernes, mais également les sociologues, ont porté une attention limitée à cette question. Or, aux prises avec le vertige d'un monde social dans lequel il est toujours possible d'agir autrement, les sociétés n'ont cessé pourtant essayer d'instaurer, sur différentes bases, des limites capables de contrer le caractère illimité de l'action humaine.

\section{Le régime économique de réalité}

Faute d'espace, laissons de côté ici les autres régimes de réalité, et centronsnous sur l'économie, véritable pivot hégémonique du régime de réalité actuel. Elle trace les limites du faisable au niveau des collectifs, associant des représentations de sens commun et une représentation hautement abstraite et savante du fonctionnement du monde social. Davantage que la science naturelle, dont elle ne cesse cependant de se réclamer, l'économie est devenue, dans sa double dimension de science sociale et de domaine de réalité, le nouveau garant des limites de la réalité. Elle remplit ainsi une fonction ontologique analogue à celle qu'a eue jadis la théologie ${ }^{18}$ ou la politique. Elle dicte l'horizon du possible et de l'impossible. Elle plie les volontés: elle ferme les débats.

Cette redoutable efficacité résulte de l'association de trois facteurs différents. D'abord, la rareté comme évidence sensible immédiate du monde (qu'il suffise de penser aux sombres annonces de Malthus); ensuite, la conception d'une mécanique économique coercitive et systémique; enfin, l'existence de sanctions (de retours de réalité) immédiats et indépassables. Sur ces points, l'accord est profond entre les analystes. Que ce soit par la main invisible des libéraux, par le Tableau de Quesnay, par le circuit keynésien, par la détermination en dernière instance chère au marxisme, le ré-encastrement de l'économie de Polanyi, l'horizon performatif de l'équilibre général ou encore l'idée d'une articulation nécessaire de facteurs institutionnels chez certains auteurs de l'école de la régulation, tous partagent, au fond, l'idée de l'existence d'un domaine social soumis à des sanctions sans équivoque. C'est cet accord qui fonde la spécificité économique du régime hégémonique de réalité contemporain. Sur ce point l'accord entre le couperet de la main invisible du marché ou les effets néfastes des rouages grippés des circuits économiques est profond.

18. François Flahault, Pourquoi limiter l'expansion du capitalisme?, Paris, Descartes \& Cie, 2003, p. 106. 
Comme jadis ont pu le faire le sacré et le profane, la hiérarchie et les statuts, l'économie institue une croyance partagée et entretenue sur ce qu'est la réalité et sur ce qui est - ou non - possible. La réussite en est d'ailleurs aussi éclatante que la nécessité à laquelle elle semble répondre. Dans le sillage du désenchantement du monde et de la fin d'un ordre moral (si Dieu est mort tout est permis) et face aux excès d'une politique animée, par le biais des révolutions, par un volontarisme sans borne (un monde dans lequel « tout est possible ${ }^{19}$ »), il revient à l'économie, dans les sociétés actuelles, d'introduire des limites, non plus en termes moraux ou hiérarchiques, mais sur des critères exclusivement factuels. Peu de choses sont devenues aussi évidentes dans les sociétés actuelles que la croyance sur les effets inévitables et inéluctables de certaines politiques économiques. Depuis quelques décennies, un acronyme à grand succès, massivement évoqué par des responsables politiques de tout bord, résume bien cette équation: TINA (There is no alternative). L'économie-comme-réalité s'institue grâce à un travail de va-et-vient permanent entre formes vulgarisées du discours économique d'une part et la production de la science économique d'autre part. Ce lien, qui n'est pas seulement idéologique, est au fondement de la fonction spécifique de l'économie dans sa capacité à tracer aujourd'hui l'impossible dans la société.

Certes, l'intronisation de l'économie comme régime hégémonique de réalité vers la fin du XVIII ${ }^{\mathrm{e}}$ siècle a connu des périodes plus volontaristes et davantage favorables à l'intervention politique que celles que nous connaissons aujourd'hui. Certes, l'économie-comme-réalité est désormais concurrencée par un régime écologique de réalité en gestation qui, déjà, est en train de transformer d'indéniables contraintes écologiques (réchauffement climatique, épuisement des matières premières) dans des limites et des seuils «infranchissables ${ }^{20}$ », alors que les contraintes écologiques ouvrent, tout autant que les contraintes économiques (ou politiques), à un conflit de stratégies. Cependant, malgré cela, l'économie a toujours une fonction décisive à l'heure de dicter le possible et l'impossible. Ce sont les faits économiques qui tracent - qui dictent -, ce que les sociétés actuelles peuvent, au nom de la réalité, se permettre.

Or, s'il est ô combien légitime de chercher des mécanismes de type causal (ou de corrélations) entre les phénomènes économiques ou sociaux, en revanche, ces constructions intellectuelles ne peuvent jamais cerner entièrement l'irréductible contingence (la non-nécessité) des faits humains. C'est

19. Hannah Arendt, Le système totalitaire, Paris, Seuil, 2005 [1951].

20. Hans Jonas, Le principe de responsabilité, Paris, Flammarion, 2009 [1979] : Jared Diamond, Effondrement, Paris, Gallimard, 2006 [2005] : Jean-Pierre Dupuy, Pour un catrastrophisme éclairé, Paris, Seuil, 2002. 
bien ce court-circuitage, la transformation des contraintes économiques en limites imaginaires, qui fonde justement l'économie-comme-réalité. Un processus qui a plus d'un point commun avec la manière dont jadis les limites furent imposées au nom des entités invisibles ou des hiérarchies politiques naturalisées.

Il est absurde de nier une certaine systématicité de l'économie: en revanche, la prétendue immédiateté des sanctions économiques doit constamment être mise sous surveillance, tant l'économie regorge de phénomènes élastiques. C'est un aspect bien visible, et bien connu d'ailleurs, à propos des crises boursières, des épisodes d'inflation, de surendettement, de la fixation des prix ou à propos de la diffusion hautement complexe des phénomènes financiers globalisés au niveau des entreprises, des régions ou des pays. Pourtant, ces constants empiriques et l'élasticité des retours de réalité, dont il est question, n'ébranlent guère la croyance dans l'imaginaire économique de la mécanique.

La dernière affirmation peut susciter de l'incrédulité. N'est-il pas vrai que le domaine économique abonde en exemples de coercitions actives? Le fait, par exemple, que l'activité du capitaliste soit déterminée, comme l'a affirmé Marx, par une logique d'accumulation qui s'impose à lui de l'extérieur, de manière objective quelle que soit sa volonté, n'en est-il pas une preuve évidente? À propos du développement des pays émergents, est-il raisonnable d'ignorer le rôle objectif des déficits chroniques de la balance des paiements, ou du poids de la dette extérieure, ou encore, de la difficulté à obtenir des crédits? Peut-on ignorer les contraintes ressenties par l'action politique à cause, par exemple, de l'étranglement de l'économie à la suite de la rareté des devises? Certes. Cependant, ces coercitions objectives se révèlent toujours, après examen, souvent bien plus labiles que ne le suggèrent ces illustrations. Si l'idée qu'il y a des domaines soumis à de fortes coercitions systémiques paraît plausible à un niveau de haute généralité, l'étude détaillée décèle toujours, y compris dans les lois d'airain de l'économie, de nombreux cas où leur fonctionnement se révèle problématique ${ }^{21}$.

Soit. Mais peut-on accepter l'idée que l'économie est le régime hégémonique de réalité contemporaine sans tenir compte du fait que les citoyens ont une (très) faible connaissance autant des faits que des principes de l'économie? En fait, si les connaissances factuelles sur l'économie font souvent largement défaut aux individus, en revanche, l'économie-comme-réalité est une représentation - une croyance - largement répandue. Les sociétés contemporaines ne diffèrent guère à ce sujet de celles du passé. Les connaissances effectives que le «peuple» avait par exemple de la théologie-politique comme 
corps doctrinal instaurant la réalité ont été elles aussi jadis bien lacunaires ${ }^{22}$. Mais cela n'a jamais empêché cette doctrine d'agir en amont comme borne effective de réalité. Les connaissances économiques sont aujourd'hui bien différentes selon les publics, mais l'économie diffuse à tous le sens d'un univers soumis à des limites - et non seulement de contraintes - indépassables. Elle distille une vision hégémonique, entretenue à la fois par le discours des économistes, par les réformes légitimées comme nécessaires des gouvernements, par le sens commun (la rareté), et, paradoxalement, par une pensée contestataire qui adhère largement à la croyance du caractère inévitable des limites économiques (et progressivement écologiques).

\section{Nouvel objet, nouvelles questions}

À la base de la vie en société se trouve donc l'épineuse question, indissociablement théorique et pratique, de la dynamique entre les contraintes et les limites. Un «nouvel objet» qui invite à explorer de «nouvelles questions» et à renouveler la vocation critique de la sociologie.

1. La reconnaissance de l'élasticité foncière de la vie sociale, invite à multiplier les études, indissociablement empiriques et théoriques, afin de mieux appréhender la contingence des sanctions autant au niveau individuel que collectif. La prise en compte de la complexité des diffusions spatiales et du déroulement temporel des phénomènes sociaux devrait permettre de mieux distinguer des moments où les contraintes sanctionnent effectivement les actions humaines d'un côté et de l'autre la fonction de l'imaginaire de la limite. Par le truchement de ces études, (dont on peut espérer qu'elles se développeront dans le domaine scolaire, le travail, les souffrances psychiques, l'insécurité, l'économie), il sera question de percer le point aveugle des chocs avec la réalité dans l'analyse et la théorie sociologiques.

2. La reconnaissance de l'élasticité foncière de la vie sociale invite à mettre en œuvre une sociologie historique d'un type particulier. La sociologie a de plus en plus tendance à cantonner ses études au seul présent et lorsqu'une dimension historique plus large est prise en compte, elle ne va jamais, mise à part quelques rarissimes exceptions, au-delà de la fin du XVIII ${ }^{\mathrm{e}}$ ou du début du XIX ${ }^{e}$ siècle. Cela - faut-il le dire? - n'a jamais été la posture des classiques. Une histoire analytique des régimes de réalité apparait comme une possibilité d'étude pour revenir à ce type de préoccupations.

22. Nicholas Abercrombie, Stephen Hill, Brian Turner, The Dominant Ideology Thesis, Londres, George Allen and Unwin, 1980. 
Si l'institution des limites de la réalité, dans chaque société, a un indéniable caractère idéologique, ce serait une erreur de réduire le travail critique à cette seule dimension. À partir d'une position d'engagement pratique dans le monde, postulée comme étant commune et transhistorique à tous les êtres humains, il serait instructif de revenir sur la manière dont sont effectivement instituées les différentes limites historiques de la réalité. Concevoir comme incommensurables les différentes compositions des mondes, n'empêche pas d'établir des ponts entre les conceptions: non seulement autour du tracé de la distinction entre humains et non-humains ${ }^{23}$, mais aussi à partir des manières dont chaque constellation conçoit les chocs avec la réalité et le sens des limites. Et si cela pose de sérieux problèmes de comparaison entre cultures et sociétés, cela n'empêche pas, néanmoins, de s'interroger sur les fonctions analogues que certains domaines jouent, en tant que modalités privilégiées de choc avec la réalité, à l'intérieur de différents régimes de réalité. Tout en postulant l'existence d'une vérité transhistorique commune - c'est toujours de la résistance du monde dont il est question -, les limites de la réalité se révèlent profondément variables dans leurs expressions historiques.

Par ce truchement, la sociologie pourrait aborder une question historique, différente de celle de l'intégration sociale. L'important dans les régimes de réalité est de comprendre comment, étant donné la consistance de la vie sociale et son élasticité foncière, la réalité devient une idée régulatrice susceptible de tracer la frontière du possible et de l'impossible. À la question de savoir comment il se fait que l'humanité a pu vivre jadis, et si longtemps, dans ce qui aujourd'hui nous semble être l'erreur et la fabulation (c'est-à-dire en croyant et agissant à partir de régimes de réalité différents du nôtre), la seule réponse est de nature pratique. C'est pourquoi reconnaître que chaque régime de réalité possède des critères pluriels et divers de vérité n'est qu'une affirmation partiellement satisfaisante. C'est la nature spécifique de la relation entre l'action et la réalité, son élasticité foncière, qui en rend compte en dernière instance.

L'histoire analytique des régimes de réalité, l'étude de cette fonction particulière, transhistorique et constitutive de la vie sociale - le tracé des limites de la réalité -, est un programme de travail spécifique de sociologie historique comparée. Le fait que cette fonction ait été remplie historiquement par différents domaines, n'implique, cependant, aucun raisonnement fonctionnaliste, même adouci. Au contraire, si la fonction assurée est largement la même (institution de la limite), le fait que le tracé soit assuré par différents domaines sociaux est à la source de substantielles variations historiques. De par leurs caractéristiques intrinsèques, indépendantes de la seule

23. Philippe Descola, Par-delà nature et culture, Paris, Gallimard, 2005. 
fonction de tracé des frontières de la réalité qu'ils exercent à un moment donné, ces domaines amènent des inflexions significatives dans la manière de concevoir les liens entre l'action et la réalité, ce qui ouvre à des raisonnements à forte consonance historique.

3. Enfin, et de manière plus fondamentale, la reconnaissance de l'élasticité foncière de la vie sociale, invite à se pencher, au-delà des fonctions de contrôle qu'a jouées la réalité dans différentes périodes historiques et sur les raisons de cette attitude millénaire, sur ses spécificités actuelles. Si cette problématique est commune à toutes les sociétés, elle a pris des caractéristiques spécifiques dans la modernité. Face à la contingence du monde, le réel exerce une fascination indéniable dans les sociétés actuelles: il s'agit du dernier grand mythe de la modernité. Plus se généralise la représentation d'une histoire ou d'un monde dépourvus de Sens, plus l'appel à la réalité devient le tribunal ultime face auquel les sociétés contemporaines veulent juger du vrai et du faux, mais surtout instituer l'impossible. Si l'appel à la réalité est aujourd'hui si probant, c'est parce que, au-delà de la question de la vérité, il permet de contrer, au nom des seuls faits, le vertige d'une société soumise à l'illimitation de l'action.

L'important n'est plus - comme jadis - de dévoiler les dieux en montrant que le monde est construit par les hommes; ni seulement de comprendre les fonctions sociales spécifiques que chaque période octroie à la réalité. Il faut analyser, aussi, les raisons secrètement morales présentes dans cette attitude. À la différence donc de ce qu'ont affirmé à l'unisson, étrangement, les trois grands maittres du soupçon - Marx, Freud et Nietzsche - les actions n'échouent pas sur le butoir intangible d'un principe de réalité, mais se déploient, au contraire, au milieu d'un clair-obscur de possibilités et de contraintes, les unes et les autres indissociablement réelles et imaginaires. Pour ouvrir les possibles, il faut s'affranchir, certainement pas de la réalité, mais de sa traduction sous-problématisée sous forme de principe de réalité. En analysant de façon critique les raisons, individuelles et collectives, de la force de la croyance transhistorique dans cette fonction sociale attribuée à la réalité, il s'agira de viser à comprendre autrement, et mieux, les fondements de la vie en société. 